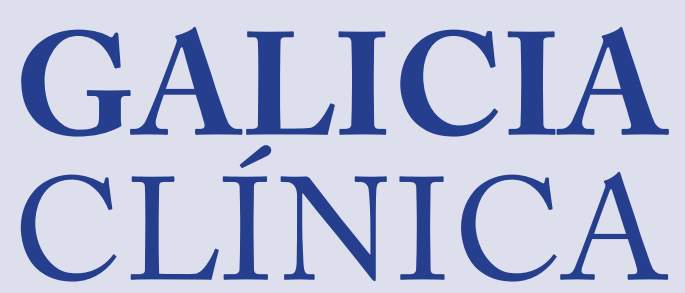

Revista oficial da Sociedade Galega de Medicina Interna (SOGAMI)

Fundada en 1929 por el Dr. Aurelio Gutiérrez Moyano

Director:

Ramón Rabuñal Rey

Directores adjuntos:

José Luis Díaz Díaz

Rafael Monte Secades

Comité editorial

Melchor Álvarez de Mon

Emilio José Casariego Vales

Alfonso Castro Beiras

María de los Ángeles Castro Iglesias

José Antonio Díaz Peromingo

Fernando Diz-Lois Martínez

Ovidio Fernández Álvarez

Ricardo Fernández Rodríguez

Carlos González Guitián

Mercedes Gutiérrez-Moyano Zimmermann

Luís Gutiérrez-Moyano Zimmermann

Fernando Antonio de la Iglesia Martínez

Pedro Mardomingo Varela

Manuel Noya García

José Domingo Pereira Andrade

Roberto Pérez Álvarez

Albino Pérez Carnero

María del Pilar Rozas Lozano

Pascual Sesma Sánchez

Antonio Torre Carballada

Comité científico

Mercedes Bal Alvaredo

Arturo Cabarcos Ortiz de Barrón

Elena Fernández Bouza

Manuel Fernández Muinelo

Javier de la Fuente Aguado

María José García Pais

Juan Antonio Garrido Sanjuán

Arturo González Quintela

José Masferrer Serra

Enrique Míguez Rey

Julio Montes Santiago

Francisco Luís Lado Lado

Victor Manuel López Mouriño

Vicente Lorenzo Zúñiga

Antonio Pose Reino

Isidro Rodríguez López

Maria Dolores Suárez Sambade

Junta Directiva de la Sogami

Presidente: Ricardo Fernández Rodríquez

Vicepresidente: Fernando Antonio De La Iglesia Martínez

Secretaria: María Del Pilar Rozas Lozano

Tesorero: José Antonio Díaz Peromingo

Vocal por La Coruña: José Luis Díaz Díaz

Vocal por Ferrol: Alida Iglesias Olleros

Vocal por Santiago: Joaquín Campos Franco

Vocal por Lugo: Juan Corredoira Sánchez

Vocal por Ourense: Juan Ignacio Telletxea Díaz

Vocal por Pontevedra: Jose María De Lis Muñoz

Vocal por Vigo: Martín Rubianes González

Vocal por 0 Barco: Antonio Eneriz Calvo

Vocal por 0 Salnés: Begoña Castro Paredes

M.I.R.: Yago Mouriño López

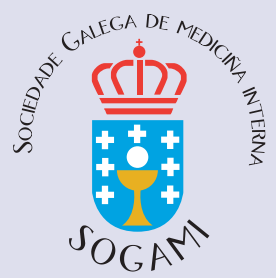

Edita: SOGAMI

ISSN: 0304-4866

Depósito legal: C 3533-2008

www.galiciaclinica.info

www.meiga.info
Presentación

\section{Reanudando una aventura}

El 15 de abril de 1929 se publicó el primer número de Galicia Clínica. En su carta de presentación se desgranaban los propósitos de la revista, que se podrían resumir en dos: por un lado poner de relieve la actividad científica realizada en Galicia, tanto desde la Universidad como desde los hospitales, clínicas particulares o cuerpos administrativos (inspectores sanitarios, forenses, etc.) y por otro contribuir al desarrollo de organizaciones de previsión que mejoraran la situación de los profesionales y sus familias. Para su máxima difusión se decidió su distribución de forma gratuita entre todos los médicos gallegos, y en la mencionada presentación se invitaba a la colaboración en la publicación a todos ellos, con una meta declarada: "aspiramos a que esta publicación, individualmente de todos y para todos, llegue a ser colectivamente el órgano de los médicos de Galicia" .

La revista fue dirigida por Aurelio Gutiérrez Moyano durante 41 años, hasta su fallecimiento el 15 de febrero de 1971 , editándose ininterrumpidamente incluso durante periodos tan dramáticos como el de la guerra civil. En esta época la revista se afianzó entre los médicos gallegos, e incluso mantuvo presencia en Iberoamérica. Posteriormente su hijo, Luís GutiérrezMoyano continuó su labor, manteniendo la revista hasta su fallecimiento. En los últimos años, por diversas causas, la revista declinó lentamente, publicándose el último número en 1996.

Ahora, con el amparo de la Sociedade Galega de Medicina Interna (SOGAMI), se ha decidido reanudar la edición de la revista. Continuamos la publicación allí donde se había dejado, por lo cual se mantiene la numeración correlativa, correspondiendo al actual el volumen 69, número 1.

Es obvio que de los objetivos originales, los aspectos profesionales tienen hoy en día sus propios cauces de representación y solución, pero creemos que todavía hay lugar para promocionar y difundir la actividad científica de los médicos gallegos. En este ámbito, siguiendo el criterio original, se mantiene la invitación a la colaboración a todos los médicos, sean internistas 0 de otras especialidades médicas. Asimismo, aunque se distribuirá de forma gratuita a los socios de la SOGAMI, podrán acceder a ella a través de nuestra página web www.galiciaclinica.info todos aquellos profesionales interesados.

Esperamos que nuestra iniciativa sea del agrado de todos y goce al menos de la misma aceptación que en su primera etapa.

Ramón Rabuñal Rey 